\title{
Manganese superoxide dismutase gene polymorphism (V16A) is associated with diabetic retinopathy in Slovene (Caucasians) type 2 diabetes patients
}

\author{
Mojca Globočnik Petrovič ${ }^{\mathrm{a}}$, Ines Cilenšek ${ }^{\mathrm{b}}$ and Daniel Petrovič ${ }^{\mathrm{b}, *}$ \\ ${ }^{a}$ Eye Clinic, University Medical Centre Ljubljana, Slovenia \\ ${ }^{\mathrm{b}}$ Institute of Histology and Embryology, Medical Faculty Ljubljana, University of Ljubljana, Slovenia
}

\begin{abstract}
Substantial data indicate that oxidative stress is involved in the development of diabetic retinopathy. Two candidate genes that affect the oxidative stress are manganese mitochondrial superoxide dismutase (Mn-SOD) and endothelial nitric oxide synthase (eNOS). The aim of the present study was to examine the role of the V16A polymorphism of the Mn-SOD gene and the $4 \mathrm{a} / \mathrm{b}$ polymorphism of the eNOS gene in the development of diabetic retinopathy in Caucasians with type 2 diabetes.

In this cross sectional case-control study 426 unrelated Slovene subjects (Caucasians) with type 2 diabetes mellitus were enrolled: 283 patients with diabetic retinopathy and the control group of 143 subjects with type 2 diabetes of duration of more than 10 years who had no clinical signs of diabetic retinopathy.

A significantly higher frequency of the VV genotype of the V16A polymorphism of the Mn-SOD was found in patients with diabetic retinopathy compared to those without diabetic retinopathy $(\mathrm{OR}=2.1,95 \% \mathrm{CI}=1.2-3.4 ; p=0.006)$, whereas the $4 \mathrm{a} / \mathrm{b}$ polymorphism of the eNOS gene failed to yield an association with diabetic retinopathy.

We may conclude that the VV genotype of the V16A polymorphism of the Mn-SOD gene was associated with diabetic retinopathy in Caucasians with type 2 diabetes, therefore it might be used as a genetic marker of diabetic retinopathy in Caucasians.
\end{abstract}

Keywords: Oxidative stress, manganese mitochondrial superoxide dismutase V16A polymorphism, 4a/b endothelial nitric oxide synthase polymorphism, genetic markers, diabetic retinopathy

\section{Introduction}

Substantial data indicate that oxidative stress is involved in the development of diabetic retinopathy $[1$, $13,15,17]$. Oxidative stress is the result of an imbalance between the amount of reactive oxygen species (ROS) and the capacity of antioxidant defense systems. Increased ROS levels can affect the redox status, and

*Corresponding author: Associate Professor Daniel Petrovẽ , MD, $\mathrm{PhD}$, Institute of Histology and Embryology, Medical Faculty Ljubljana, Korytkova 2, SI-1000 Ljubljana, Slovenia. Tel.: +386 1 5437367; Fax: +386 1 5437361; E-mail: daniel.petrovic@mf.uni-lj. si. an overall oxidative milieu tends to increase protein phosphorylation and favour cell proliferation [1]. The most common ROS in the cell is the superoxide radical, which is produced during oxidative phosphorylation within mitochondria. A key enzyme in antioxidant defence systems is manganese mitochondrial superoxide dismutase (Mn-SOD), which catalyses the removal of superoxide radicals at the site of production, the matrix side of the inner mitochondrial membrane. A number of polymorphisms in this sequence have been described, but only the A16V has been demonstrated to have a functional significance $[11,26,27]$. In fact, the protein encoded by the Val (V) allele, which disrupts the alpha -helix structure, is retained at the level of the 
mitochondrial inner membrane and has been associated with a $30-40 \%$ lower activity and increased susceptibility to oxidative stress [27]. The V16A polymorphism of the Mn-SOD gene may therefore cause interindividual differences in Mn-SOD protein localization. Additionally, large interracial differences in the allele frequency have been reported $[6,11,19,24,27]$.

Another candidate gene that affects oxidative stress is endothelial nitric oxide synthase (eNOS). Nitric oxide (NO) production has been reported to be affected by environmental and genetical factors $[8,20,23,28]$. A number of polymorphisms of the eNOS gene have been described, and a 27 base pairs (bp) repeat in the intron 4 of the eNOS gene (eNOS $4 \mathrm{a} / \mathrm{b}$ ) has been demonstrated to have a functional significance $[23,28]$. NO production has been reported to be affected by the presence of high glucose concentrations $[8,20]$. Moreover, the availability of biologically active $\mathrm{NO}$ in a given tissue depends not only on the rate of its production, but also the rate of its inactivation by superoxide $[5,12$, 21]. However, excess production of ROS determined by environmental factors (high glucose concentrations) and genetic polymorphisms results in oxidative stress, cytotoxicity and tissue damage [8,20,30,32].

The aim of the present study was to elucidate the role of the V16A polymorphism of the Mn-SOD gene and the $4 \mathrm{a} / \mathrm{b}$ polymorphism of the eNOS gene in the development of diabetic retinopathy in Caucasians with type 2 diabetes.

\section{Patients and methods}

In this cross sectional case-control study 426 unrelated Slovene subjects (Caucasians) with type 2 diabetes mellitus with a defined ophthalmologic status were enrolled. Patients were classified as having type 2 diabetes according to the current American Diabetes Association criteria [29]. Subjects were recruited from the Eye Clinic and from the Diabetic Outpatient Clinic of the University Medical Centre Ljubljana between January 2002 and April 2007.

Fundus examination was performed by a senior ophthalmologist (M.P.) after pupil dilatation (tropicamide and phenylephrine $2.5 \%$ ) using slit lamp biomicroscopy with non-contact lens, and was electronically documented with a $50^{\circ}$-angle fundus camera (Topcon-TRC 40-IX; Tokyo, Japan). Staging of diabetic retinopathy was determined according to the ETDRS retinopathy severity scale [14].
The study group consisted of 283 patients with diabetic retinopathy: 205 subjects with proliferative diabetic retinopathy (new vessel formation and/or fibrous proliferation with or without vitreous hemorrhage) and 78 subjects with non-proliferative diabetic retinopathy (microaneurysms, retinal hemorrhages, hard exudates) [14]. The control group consisted of 143 subjects with type 2 diabetes of duration of more than 10 years who had no clinical signs of diabetic retinopathy.

To avoid the confounding effect of impaired kidney function, the patients with overt nephropathy were not enrolled. The study was approved by the national medical ethics committee. After an informed consent for participation in the study was obtained, a detailed interview was made.

The V16A polymorphism of the Mn-SOD gene was evaluated by RFLP, using the primers: P1: 5'-CAG CCC AGC CTG CGT AGA CGG -3' and P2: 5'CTT GGC CAA CGC CTC CTG GTA CTT-3', and the BsaW1 restriction enzyme as described by Degoul et al. [11]. The eNOS $4 \mathrm{a} / \mathrm{b}$ gene polymorphism was analyzed as described previously $[22,33]$.

Genotyping was performed by two researchers (I.C., D.P.), blinded to the retinopathy status of the patients. Chi-square test was used to compare discrete variables. Continuous clinical data were compared by unpaired Students $t$ test. In addition, all variables that showed significant differences by univariate methods (chi-square test, unpaired Students $t$ test) were analysed together in a logistic regression analysis.

Assuming the significance level of 0.05 , frequency of risk allele of 0.161 in the control group and of 0.283 in the retinopathy group, we calculated the power of our study sample (283 cases, 143 controls) to be $80 \%$. Statistical analysis was performed using the SPSS program for Windows version 14 (SPSS Inc. Illinois).

\section{Results}

The characteristics of the cases and control subjects are listed in Table 1. Cases had earlier onset of diabetes and longer duration of type 2 diabetes compared to the diabetics without diabetic retinopathy. Additionally they had higher incidence of insulin herapy than the controls (diabetics without diabetic retinopathy). Additionally they had lower total and HDL cholesterol levels than the controls. There were no significant differences in hypertension, smoking, LDL cholesterol levels and triglyceride levels between the cases and control subjects. 
Table 1

Characteristics of patients with diabetic retinopathy (cases) and patients without diabetic retinopathy (controls)

\begin{tabular}{lccc}
\hline Characteristics & Cases n $(\%)$ & Controls n $(\%)$ & $\mathrm{P}$ \\
\hline Number & 283 & 143 & \\
Age (years) & $66.0 \pm 9.5$ & $66.9 \pm 11.5$ & 0.4 \\
Male sex (\%) & $133(47.0)$ & $57(39.9)$ & 0.2 \\
Duration of diabetes (years) & $19.6 \pm 8.9$ & $16.5 \pm 6.6$ & 0.001 \\
Patients on insulin therapy (\%) & $192(67.8)$ & $66(46.1)$ & $<0.001$ \\
Age of diabetes onset & $46.5 \pm 11.3$ & $53.3 \pm 12.1$ & $<0.001$ \\
HbA $_{1 c}(\%)$ & $8.0 \pm 1.6$ & $8.2 \pm 1.6$ & 0.1 \\
Systolic blood pressure $(\mathrm{mm} \mathrm{Hg)}$ & $144 \pm 22$ & $145 \pm 20$ & 0.9 \\
Diastolic blood pressure $(\mathrm{mm} \mathrm{Hg)}$ & $84 \pm 10$ & $84 \pm 9$ & 0.7 \\
BMI (kg/m $\left.{ }^{2}\right)$ & $27.9 \pm 4.6$ & $27.7 \pm 4.4$ & 0.9 \\
History of hypertension $(\%)$ & $206(72.8)$ & $100(70)$ & 0.6 \\
Smokers $(\%)$ & $31(11.0)$ & $15(10.5)$ & 0.4 \\
Total cholesterol (mmol/l) & $5.4 \pm 1.2$ & $5.5 \pm 1.2$ & 0.1 \\
HDL cholesterol (mmol/l) & $1.1 \pm 0.4$ & $1.2 \pm 0.4$ & 0.1 \\
LDL cholesterol (mmol/l) & $3.1 \pm 1.0$ & $3.2 \pm 0.9$ & 0.2 \\
Triglycerides (mmol/l) & $2.2 \pm 1.3$ & $2.6 \pm 1.9$ & 0.1 \\
\hline
\end{tabular}

Table 2

Distribution of Mn-SOD and eNOS genotypes/alleles in patients with diabetic retinopathy (cases) and in those without diabetic retinopathy (controls)

\begin{tabular}{|c|c|c|c|c|}
\hline Genotype/allele & Cases n (\%) & Controls n (\%) & $\mathrm{P}$ & $\begin{array}{l}\text { Odds ratio ( } 95 \% \\
\text { confidence interval) }\end{array}$ \\
\hline \multicolumn{5}{|l|}{ MnSOD } \\
\hline AA genotype (wild type) & $63(22.3)$ & $51(35.7)$ & & 1 \\
\hline $\mathrm{AV}$ genotype & $140(49.5)$ & $69(48.3)$ & $0.037^{1}$ & $1.6(1.0-2.6)^{1}$ \\
\hline VV genotype & $80(28.3)$ & $23(16.1)$ & $0.001^{2}$ & $2.8(1.6-5.1)^{2}$ \\
\hline \multirow[t]{2}{*}{ VV or AV genotype } & & & $0.006^{3}$ & $2.1(1.2-3.4)^{3}$ \\
\hline & 283 & 143 & & \\
\hline $\mathrm{V}$ allele & $300(53.0)$ & $115(40.2)$ & $0.004^{4}$ & \\
\hline A allele & $266(47.0)$ & $171(59.8)$ & & \\
\hline \multicolumn{5}{|l|}{ eNOS } \\
\hline bb genotype (wild type) & $176(62.2)$ & $92(64.3)$ & & 1 \\
\hline ab genotype & $91(32.2)$ & $44(30.8)$ & $0.8^{5}$ & $1.05(0.6-1.6)^{5}$ \\
\hline \multirow[t]{3}{*}{ aa genotype } & $16(5.6)$ & $7(4.9)$ & $0.9^{6}$ & $1.2(0.9-1.4)^{6}$ \\
\hline & & & $0.6^{7}$ & $1.1(0.3-3.7)^{7}$ \\
\hline & 283 & 143 & & \\
\hline a allele & $123(21.7)$ & $58(20.3)$ & $0.6^{8}$ & \\
\hline $\mathrm{b}$ allele & $443(78.3)$ & $228(79.7)$ & & \\
\hline
\end{tabular}

${ }^{1} \mathrm{P}$-value and OR (AV versus AA), ${ }^{2} \mathrm{P}$-value and $\mathrm{OR}$ (VV versus AA), ${ }^{3} \mathrm{P}$-value and OR (VV versus AV plus AA), ${ }^{4} \mathrm{P}$ value for allele frequency, ${ }^{5} \mathrm{P}$-value and $\mathrm{OR}$ (ab versus bb), ${ }^{6} \mathrm{P}$-value and OR (aa versus bb), ${ }^{7} \mathrm{P}$-value and OR (aa versus ab plus bb), ${ }^{8} \mathrm{P}$ value for allele frequency.

The V16A Mn-SOD genotype distribution and the $4 \mathrm{a} / \mathrm{b}$ eNOS genotype distribution in cases and controls did not depart from Hardy-Weinberg equilibrium significantly (V16A: cases $\chi^{2}=0.014, p=0.91$; controls $\chi^{2}=0.002, p=0.97 ; 4 \mathrm{a} / \mathrm{b}$ eNOS: cases $\chi^{2}=0.85, p=0.35$; controls $\chi^{2}=0.55, p=0.56$ ). The frequencies of the genotypes of the V16A polymorphism of the Mn-SOD gene and of the $4 \mathrm{a} / \mathrm{b}$ polymorphism of the eNOS gene are shown in Table 2; a significantly higher frequency of the Val/Val (VV) genotype was found in patients with diabetic retinopathy compared to subjects without diabetic retinopathy $(\mathrm{OR}=2.1,95 \% \mathrm{CI}=1.2-3.4 ; p=0.006)$.
We compared the frequency of genotypes and alleles between cases and controls (Table 2). There was no significant difference in the frequency of $4 \mathrm{a} / \mathrm{b}$ eNOS genotypes and alleles between the two groups.

The variables that showed significant differences by chi-square test and unpaired Students $t$ test (the VV genotype of the V16A polymorphism, insulin therapy, duration of diabetes, age of onset of diabetes) were analysed together in a logistic regression analysis. In the logistic regression models ((first model - VV genotype vs. AA plus AV genotypes; second model - VV genotype vs. AA genotype) the VV genotype of the V16A polymorphism was independent risk factors for 
Table 3

Adjusted odds ratios for risk factors for diabetic retinopathy.

\begin{tabular}{lll}
\hline Risk factors & Odds ratio (95\% CI) & $\mathrm{P}$ \\
\hline VV genotype $^{1}$ & $1.8(1.0-3.2)$ & 0.035 \\
Insulin therapy & $2.5(1.5-4.0)$ & $<0.001$ \\
age of onset of diabetes & $1.041(1.015-1.067)$ & 0.002 \\
Duration of diabetes & $0.994(0.96-1.029)$ & 0.7 \\
\hline
\end{tabular}

${ }^{1}$ The V16A polymorphism of the Mn-SOD gene.

diabetic retinopathy, whereas the AV genotype was not an independent risk factor for diabetic retinopathy (Table 3 ).

\section{Discussion}

The present study provides evidence that the $\mathrm{VV}$ genotype of the V16A polymorphism of the Mn-SOD gene might be a risk factor for diabetic retinopathy in the Slovene population (Caucasians) with type 2 diabetes, whereas the $4 \mathrm{a} / \mathrm{b}$ polymorphism of the eNOS gene is not. Our findings are in accordance with some reports demonstrating an association of the V16A polymorphism of the Mn-SOD gene with microvascular complications of diabetes [6,24]. Nomiyama et al. [24] have reported an association between the V16A polymorphism of the Mn-SOD gene and diabetic nephropathy in Japanese type 2 diabetic patients. The frequency of the VV genotype was significantly higher in patients with diabetic nephropathy $(84.1 \%$ in subjects with macroalbuminuria and $84.8 \%$ in subjects with microalbuminuria) in comparison with subjects with normoalbuminuria (70.8\%). Chistyakov et al. [6] have reported an association between the VV genotype of the V16A polymorphism and diabetic neuropathy $(15.9 \%$ in subjects with neuropathy and $2.4 \%$ in subjects without neuropathy), whereas the Mn-SOD allele frequency did not differ between Russian type 1 diabetic and nondiabetic subjects. Strokov et al. [34] have also reported an association between the VV genotype of the V16A polymorphism and diabetic neuropathy in Russian type 1 diabetic and nondiabetic subjects $(20.3 \%$ in subjects with neuropathy and $5.5 \%$ in subjects without neuropathy). In a very recent report the V16A polymorphism of the Mn-SOD gene has been associated with diabetic macular edema in Korean type 2 diabetic patients, but not with either the development of diabetic retinopathy or the progression of diabetic retinopathy [19].

The retina is particularly susceptible to oxidative stress because of its high consumption of oxygen, high proportion of polyunsaturated fatty acids, and exposure to visible light [3]. Oxidative stress can influ- ence the expression of multiple genes, including signalling molecules; overexpression of these genes may cause mitochondrial dysfunction and peroxidization of the lipid and protein structure, which induce a variety of cellular dysfunctions leading to retinopathy [7, 16,18]. Kowluru et al. [17] have recently investigated the effect of overexpression of the Mn-SOD gene on glucose-induced retinal endothelial cell oxidative stress, nitrosative stress, and apoptosis. They have demonstrated that Mn-SOD over-expression prevented a glucose-induced increase in oxidative stress and apoptosis of retinal endothelial cells suggesting a protective role of Mn-SOD in the pathogenesis of diabetic retinopathy [17]. Additionally, large inter-racial differences in the allele frequency have been reported so far, and only few studies enrolled general population in Caucasians $[4,6,11,19,24,31]$. The frequency of the $\mathrm{V}$ allele in general population is around 0.50 in Caucasians [4,6,31].

Plasma nitric oxide metabolite levels of subjects with the aa genotype of the $4 \mathrm{a} / \mathrm{b}$ eNOS polymorphism are significantly lower when compared with those of individuals without the aa genotype (ab genotype plus bb genotype) [30]. In the study, however, we did not find an association between the aa genotype of the $4 \mathrm{a} / \mathrm{b}$ eNOS polymorphism and diabetic retinopathy. The results of our study are in accordande with a casecontrol asociation study performed in Japanese population and in Caucasian population [2,28]. In both studies they failed to demonstrate an association between the aa genotype and and diabetic retinopathy $[2,28]$. In French study, however, an association between the bb genotype and diabetic retinopathy was reported $[2,28]$.

Our results increase the knowledge of genetic risk factors for developing diabetic retinopathy in type 2 diabetes. It seems, however, that the retinopathy cases had more severe form of type 2 diabetes in comparison with diabetics without diabetic retinopathy, i.e. cases had earlier onset of diabetes, longer duration of type 2 diabetes and higher incidence of insulin herapy compared to the diabetics without diabetic retinopathy. Our findings are in accordance with UKPDS 22 and 50 studies that demonstrated importance of earlier onset of diabetes and longer duration diabetes [35,36]. Moreover, insulin therapy was independently of the V16A gene polymorphism statistically significantly associated with diabetic retinopathy. This finding suggests the existence of other factors such as differences in the ability of insulin secretion, or differences in the frequency of episodes of hypoglycemia, or adverse events associated with insulin therapy (hypoglycemia, worsening 
diabetic retinopathy if $\mathrm{HbA}_{1 c}$ decreases rapidly) $[9,10$, 25].

Further studies enrolling larger numbers of patients from different populations are needed to confirm our findings. Namely, genetic association studies are prone to beta statistical error and population-specific genotype effects, all of which make the results difficult to reproduce. In fact, the calculated power of our study for the MnSOD polymorphism was $80 \%$ taking into account the size of our study sample, and the frequencies of risk allele in cases and controls.

We may conclude that the VV genotype of the V16A polymorphism of the Mn-SOD gene might be a risk factor for diabetic retinopathy in the Slovene population (Caucasians) with type 2 diabetes and may be used as a genetic marker of diabetic retinopathy in Caucasians. Understanding the role of the Mn-SOD gene to modify the course of retinopathy could elucidate important molecular targets for future pharmacological interventions [17].

\section{Acknowledgements}

The authors thank Ms Mojca Pirc, BA, for revising the English.

\section{References}

[1] A.G. Allen and M. Tresini, Oxidative stress and gene regulation, Free Radic Biol Med 28 (2000), 463-499.

[2] T. Awata T, T. Neda, H. Iizuka, S. Kurihara, T. Ohkubo, N. Takata, M. Osaki, M. Watanabe, Y. Nakashima, T. Sawa, K. Inukai, I. Inoue, M. Shibuya, K. Mori, S. Yoneya and S. Katayama, Endothelial nitric oxide synthase gene is associated with diabetic macular edema in type 2 diabetes, Diabetes Care 27 (2004), 2184-2190.

[3] S. Beatty, H. Koh, M. Phil, D. Henson and M. Boulton, The role of oxidative stress in the pathogenesis of agerelated macular degeneration, Surv Ophthalmol 45 (2000), 115-134.

[4] M. Bergman, M. Ahnstrom, P. Palmback Wegman and S. Wingren, Polymorphism in the manganese superoxide dismutase (MnSOD) gene and risk of breast cancer in young women, J Cancer Res Clin Oncol 131 (2005), 39-44.

[5] V.M. Campese, R.K. Sindhu, S. Ye, Y. Bai, N.D. Vaziri and B. Jabbari, Regional expression of NO synthase, NAD(P)H oxidase and superoxide dismutase in the rat brain, Brain Res 1134 (2007), 27-32.

[6] D.A. Chistyakov, K.V. Savost'anov, E.V. Zotova and V.V. Nosikov, Polymorphisms in the Mn-SOD and EC-SOD genes and their relationship to diabetic neuropathy in type 1 diabetes mellitus, BMC Med Genet 2 (2001), 4-10.

[7] C. Cingolani, B. Rogers, L. Lu, S. Kachi, J. Shen and P.A. Campochiaro, Retinal degeneration from oxidative damage, Free Radical Biol Med 40 (2006), 660-669.
[8] M. Colasanti and H. Suzuki, The dual personality of NO, Trends Pharmacol Sci 21 (2000), 249-252.

[9] K. Dahl-Jorgensen, O. Brinchmann-Hansen, K.F. Hanssen, L. Sandvik and O. Aagenaes, Rapid tightening of blood glucose control leads to transient deterioration of retinopathy in insulin dependent diabetes mellitus: the Oslo study, $\mathrm{Br}$ Med $\mathrm{J} 290$ (1985), 811-815.

[10] D.E. DeWitt and I.B. Hirsch, Outpatient insulin therapy in type 1 and type 2 diabetes mellitus: scientific review, JAMA 289 (2003), 2254-2264.

[11] F. Degoul, A. Sutton, A. Mansouri, C. Cepanec, C. Degott, B. Fromenty, M. Beaugrand and D. Valla, Homozygosity for alanine in the mitochondrial targeting sequence of superoxide dismutase and risk for severe alcoholic liver disease, Gastroenterology 120 (2001), 1468-1474.

[12] H. Ding, M. Hashem and C. Triggle, Increased oxidative stress in the streptozotocin-induced diabetic apoE-deficient mouse: Changes in expression of NADPH oxidase subunits and eNOS, Eur J Pharmacol 561 (2007), 121-128.

[13] Y. Du, C.M. Miller and T.S. Kern, Hyperglycemia increases mitochondrial superoxide in retina and retinal cells, 35 Free Radical Biol Med (2003), 1491-1499.

[14] Early Treatment Diabetic Retinopathy Study Research Group: Grading diabetic retinopathy from stereoscopic color fundus photographs-an extension of the modified Airlie House classification ETDRS report number 10, Ophthalmology 98(Suppl 5) (1991), 786-806.

[15] S.K. Jain, R. McVie, J. Duett and J.J. Herbst, Erythrocyte membrane lipid peroxidation and glycosylated hemoglobin in diabetes, Diabetes 38 (1989), 1539-1543.

[16] K.Kannan and S.K. Jain, Oxidative stress and apoptosis, Pathophysiology 7 (2000), 153-163.

[17] R.A. Kowluru, L. Lamia Atasi and Y.S. Ho, Role of Mitochondrial Superoxide Dismutase in the Development of Diabetic Retinopathy Invest Ophthalmol, Vis Sci 47 (2006), 1594-1599.

[18] R.A. Kowluru, Diabetic retinopathy: mitochondrial dysfunction and retinal capillary cell death, Antioxid Redox Signaling 7 (2005), 1581-1587.

[19] S.J. Lee and M.G. Choi, Association of manganese superoxide dismutase gene polymorphism (V16A) with diabetic macular edema in Korean type 2 diabetic patients, Metabolism $\mathbf{5 5}$ (2006), 1681-1688.

[20] H. Li and U. Forstermann, Nitric oxide in the pathogenesis of vascular disease, J Pathol 190 (2000), 244-254.

[21] P.A. Marsden, H.H. Heng, S.W. Scherer, R.J. Stewart, A.V. Hall, X.M. Shi, L.C. Tsui and K.T. Schappert, Structure and chromosomal localization of the human constitutive endothelial nitric oxide synthase gene, J Biol Chem 268 (1993), 17478-17488.

[22] A. Milutinovic and H. Hruskovicova, The eNOS gene polymorphism does not have a major impact on lipid parameters and premature coronary artery disease in Slovene men (Caucasians), Folia Biol (Praha) 51 (2005), 47-49.

[23] S. Neugebauer, T. Baba and T. Watanabe, Association of the nitric oxide synthase gene polymorphism with an increased risk for progression to diabetic nephropathy in type 2 diabetes, Diabetes 49 (2000), 500-503.

[24] T. Nomiyama, Y. Tanaka, L. Piao, K. Nagasaka, K. Sakai, T. Ogihara, K. Nakajima, H. Watada and R. Kawamori, The polymorphism of manganese superoxide dismutase is associated with diabetic nephropathy in Japanese type 2 diabetic patients, J Hum Genet 48 (2003), 138-141.

[25] V. Poulaki, W. Qin, A.M. Joussen, P. Hurlbut, S.J. Wiegand, J. Rudge, G.D. Yancopoulos and A.P. Adamis, Acute inten- 
sive insulin therapy exacerbates diabetic blood-retinal barrier breakdown via hypoxia-inducible factor-1 and VEGF, $J$ Clin Invest 109 (2002), 805-815.

[26] S. Shimoda-Matsubayashi, H. Matsumine, T. Kobayashi, Y. Nakagawa-Hattori, Y. Shimizu and Y. Mizuno, Structural dimorphism in the mitochondrial targeting sequence in the human manganese superoxide dismutase gene. A predictive evidence for conformational change to influence mitochondrial transport and a study of allelic association in Parkinson (http://www.springerlink.com/content/ku64v3704545x51p/ xlarge8217.gif) isn't in documents disease, Biochem Biophys Res Commun 13 (1996), 561-565.

[27] A. Sutton, A. Imbert, A. Igoudjil, V. Descatoire, S. Cazanave, D. Pessayre and F. Degoul, The manganese superoxide dismutase Ala16Val dimorphism modulates both mitochondrial import and mRNA stability, Pharmacogenet Genomics 15 (2005), 311-319.

[28] M.J. Taverna, A. Sola, C. Guyot-Argenton, N. Pacher, F. Bruzzo, A. Chevalier, G. Slama, G. Reach and J.L. Selam, The eNOS4 polymorphism of the endothelial nitric oxide synthase predicts risk for severe diabetic retinopathy, Diabet Med 19 (2002), 240-245.

[29] The Expert Committee on the Diagnosis and Classification of Diabetes Mellitus, Report of the Expert Committee on the Diagnosis and Classification of Diabetes Mellitus, Diabetes Care 20 (1997), 1183-1197.

[30] T. Tsukada, K. Yokoyama, T. Arai, F. Takemoto, S. Hara, A. Yamada, Y. Kawaguchi, T. Hosoya and J. Igari, Evidence of association of the ecNOS gene polymorphism with plasma NO metabolite levels in humans, Biochem Biophys Res Commun 245 (1998), 190-193.

[31] L. Valenti, D. Conte, A. Piperno, P. Dongiovanni, A.L. Fracanzani, M. Fraquelli, V. Vergani, C. Gianni, L. Carmagnola and S. Fargion, The mitochondrial superoxide dismutase A16V polymorphism in the cardiomyopathy associated with hereditary haemochromatosis, J Med Genet 41 (2004), 46-50.

[32] N.D. Vaziri, Y.S. YS, C.Y. Lin, V.W. Lin and R.K. Sindhu, NAD $(\mathrm{P}) \mathrm{H}$ oxidase, superoxide dismutase, catalase, glutathione peroxidase and nitric oxide synthase expression in subacute spinal cord injury, Brain Res (2004), 76-83.

[33] X.L.Wang, A.S. Sim, R.F. Badenhop, R.M. McCredie and D.E. Wilcken, A smoking-dependent risk of coronary artery disease associated with a polymorphism of the endothelial nitric oxide synthase gene, Nature Med 2 (1996), 41-45.

[34] I.A. Strokov, T.R. Bursa, O.I. Drepa, E.V. Zotova, V.V. Nosikov and A.S. Ametov, Predisposing genetic factors for diabetic polyneuropathy in patients with type 1 diabetes: a population-based case-control study, Acta Diabetol 40(Suppl 2) (2003), S375-S379.

[35] T.M. Davis, I.M. Stratton, C.J. Fox, R.R. Holman and R.C. Turner, U.K. Prospective Diabetes Study 22. Effect of age at diagnosis on diabetic tissue damage during the first 6 years of NIDDM, Diabetes Care 20 (1997), 1435-1441.

[36] I.M. Stratton, E.M. Kohner, S.J. Aldington, R.C. Turner, R.R. Holman, S.E. Manley and D.R. Matthews, UKPDS 50: risk factors for incidence and progression of retinopathy in Type II diabetes over 6 years from diagnosis, Diabetologia 44 (2001), 156-163. 


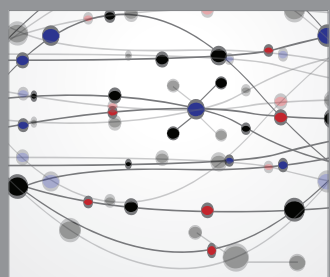

The Scientific World Journal
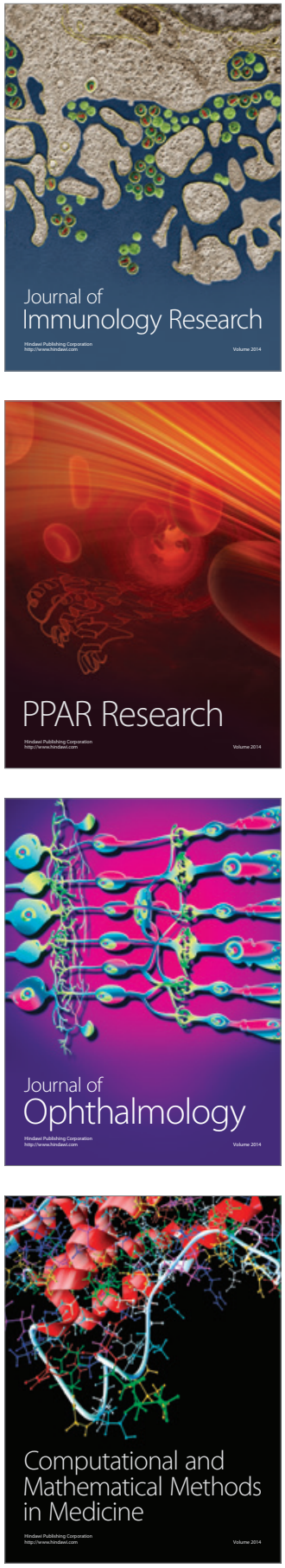

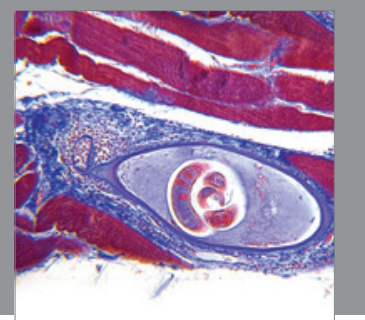

Gastroenterology

Research and Practice
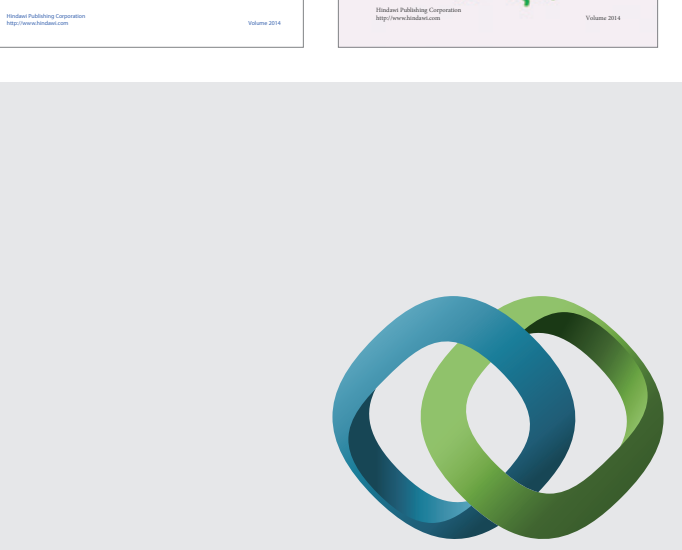

\section{Hindawi}

Submit your manuscripts at

http://www.hindawi.com
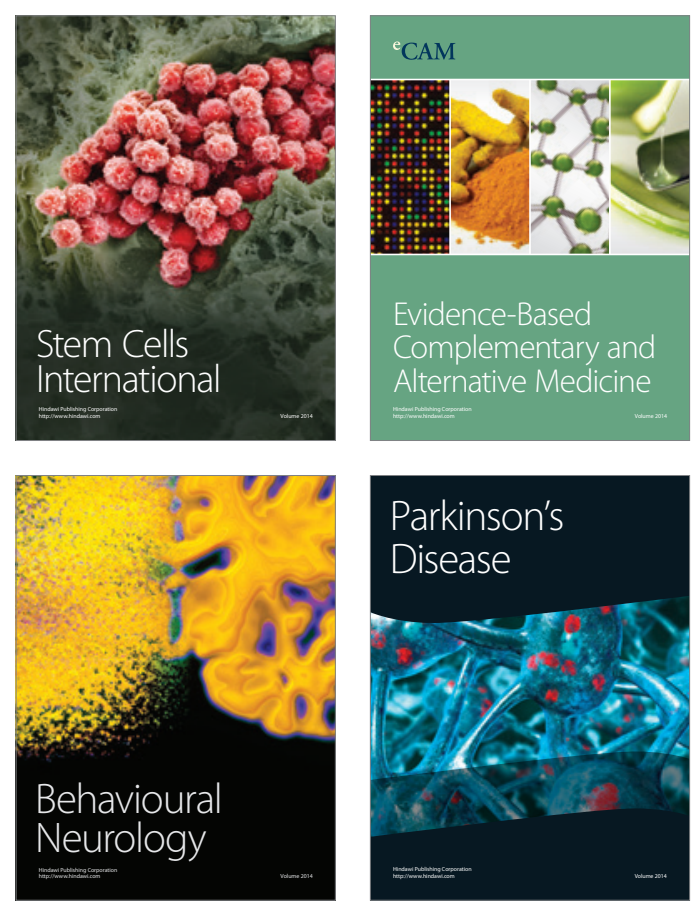

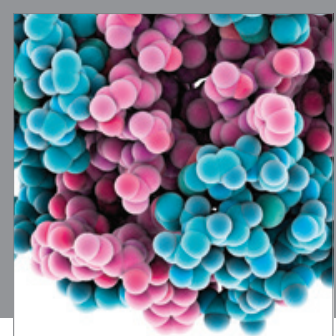

Journal of
Diabetes Research

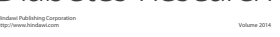

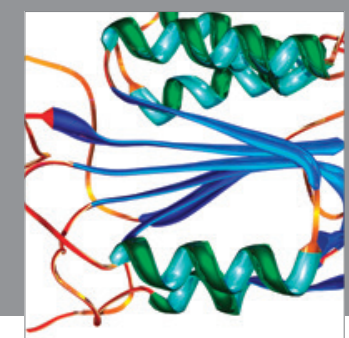

Disease Markers
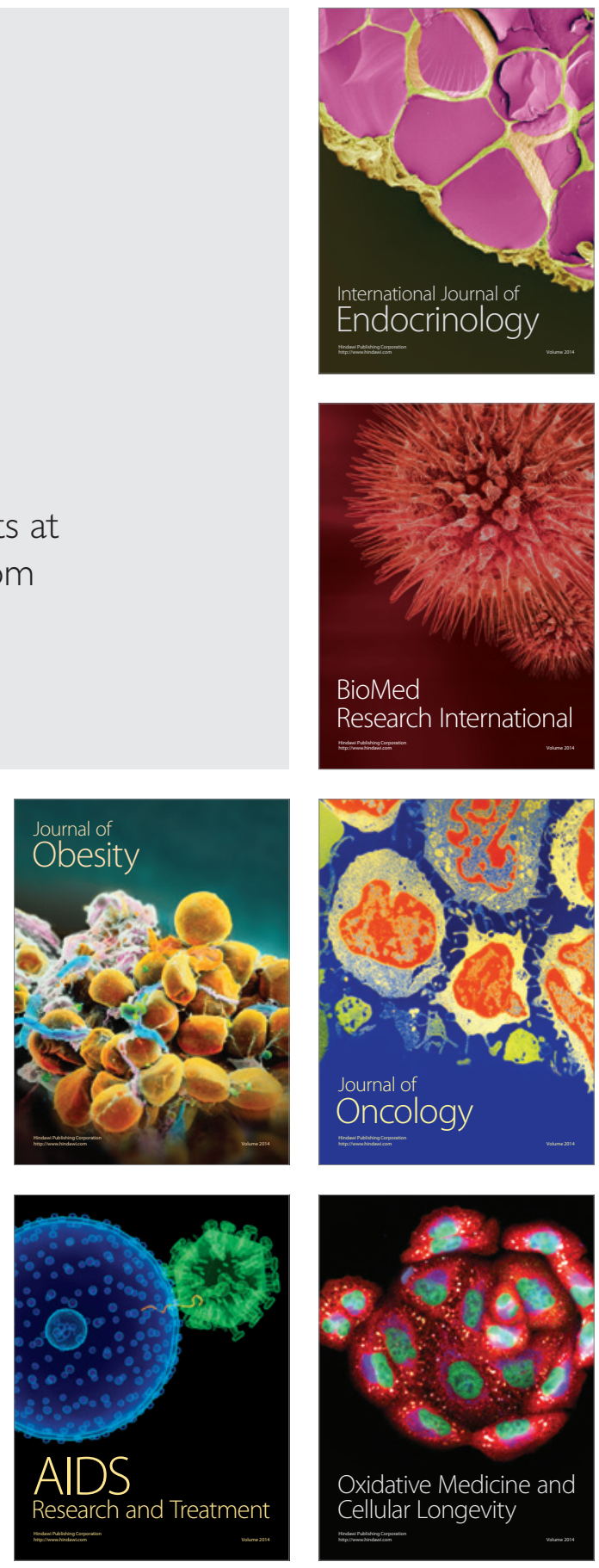